\title{
Leg and arm adiposity is inversely associated with diastolic hypertension in young and middle-aged United States adults
}

Aayush Visaria ${ }^{1,2^{*}}$ (D) David Lo ${ }^{2}$, Pranay Maniar ${ }^{2,3}$, Bhoomi Dave ${ }^{3}$ and Parag Joshi ${ }^{4}$ (D)

\begin{abstract}
Background: We sought to determine the association between appendicular adiposity and hypertension, with the purpose of better understanding the role of body fat distribution on blood pressure (BP).

Methods: We included 7411 adults aged 20 to 59 who were not taking antihypertensives and without cardiovascular disease from the 2011 to 2018 National Health and Nutrition Examination Surveys. Leg \& arm adiposity, determined via dual-energy X-ray absorptiometry scans, was defined as percent of total body fat present in legs/arms (leg/total\%, arm/total\%). Measures were categorized into sex-specific tertiles. We estimated change in BP and odds ratios (ORs) of hypertension (BP $\geq 130 / 80$ ) and hypertension subtypes using multivariable, survey design-adjusted linear \& logistic regression, respectively.
\end{abstract}

Results: Of the participants, 49\% were female, the average (standard deviation) age was 37.4 (0.3) years, and 24\% had hypertension. Those in the highest tertile (T3) of leg/total\% had 30\% decreased adjusted ORs (aOR) of hypertension compared to the lowest tertile ( $\mathrm{T} 1$; aOR, 0.70; $95 \%$ confidence interval $[95 \% \mathrm{Cl}], 0.55-0.89)$. This association was not significant for arm/total\% (0.89, 0.68-1.17). T3 of leg/total\% was associated with $49 \%$ lower, $41 \%$ lower, and unchanged relative odds of isolated diastolic hypertension (IDH), systolic-diastolic hypertension (SDH), and isolated systolic hypertension (ISH) compared to T1 (IDH: 0.51, 0.37-0.70; SDH: 0.59, 0.43-0.80; ISH: 1.06, 0.701.59). For every $10 \%$ increase in leg/total\%, diastolic BP decreased by an adjusted mean $3.5 \mathrm{mmHg}(95 \% \mathrm{Cl},-4.8$ to - 2.2) in males and $1.8 \mathrm{mmHg}(95 \% \mathrm{Cl},-2.8$ to -0.8$)$ in females ( $P<0.001$ for both).

Conclusions: A greater proportional distribution of fat around the legs is inversely, independently associated with hypertension, and more specifically, diastolic hypertension (IDH and SDH).

Keywords: Body composition, Body fat distribution, Hypertension, Epidemiology, Blood pressure

\footnotetext{
* Correspondence: aayush.visaria@rutgers.edu

Study, in part, was presented at the American Heart Association 2020 Hypertension Sessions.

'Department of Medicine, Rutgers New Jersey Medical School, Newark, NJ,

USA

${ }^{2}$ North American Disease Intervention, Rutgers University, New Brunswick, NJ,

USA

Full list of author information is available at the end of the article
}

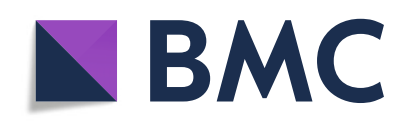

() The Author(s). 2021 Open Access This article is licensed under a Creative Commons Attribution 4.0 International License, which permits use, sharing, adaptation, distribution and reproduction in any medium or format, as long as you give appropriate credit to the original author(s) and the source, provide a link to the Creative Commons licence, and indicate if changes were made. The images or other third party material in this article are included in the article's Creative Commons licence, unless indicated otherwise in a credit line to the material. If material is not included in the article's Creative Commons licence and your intended use is not permitted by statutory regulation or exceeds the permitted use, you will need to obtain permission directly from the copyright holder. To view a copy of this licence, visit http://creativecommons.org/licenses/by/4.0/ The Creative Commons Public Domain Dedication waiver (http://creativecommons.org/publicdomain/zero/1.0/) applies to the data made available in this article, unless otherwise stated in a credit line to the data. 


\section{Background}

Hypertension is a common chronic illness in the United States, with prevalence estimates greater than 100 million patients $[1,2]$. There are several well-established risk factors for hypertension, including obesity, physical inactivity, high salt diet, and stress [2-6]. In addition, studies have demonstrated that hypertension subtypes (isolated diastolic hypertension, IDH; systolic-diastolic hypertension, SDH; isolated systolic hypertension, ISH) have unique age and demographic distributions and differences in significance of traditional risk factors $[7,8]$. For example, elevated body mass index (BMI) was more significantly associated with IDH than either ISH or SDH in US adults [9].

While obesity has been associated with hypertension and downstream cardiovascular complications through mechanisms such as activation of the renin-angiotensinaldosterone axis, increased cardiac output, sympathetic activation, insulin resistance, and adipocyte-related hormone release $[10,11]$, recent literature has provided evidence that distribution of body fat is just as, if not more, important than total body adiposity. Total body adiposity can be divided into appendicular adiposity (arm \& leg adiposity), truncal adiposity, and head \& neck adiposity. Truncal, visceral adiposity, often estimated by waist circumference, has been consistently positively associated with cardiometabolic conditions [11, 12]. Leg adiposity, on the other hand, has been inversely associated with cardiometabolic factors such as insulin resistance [1317], metabolic syndrome [18-20], and cardiovascular disease \& mortality [21-26] in several cross-sectional studies, albeit of questionable clinical significance. Additionally, the majority of leg adiposity is subcutaneous fat, which has been shown to be metabolically distinct to visceral adiposity. Subcutaneous fat is less likely to release sympathetic activating or renin-angiotensinaldosterone axis activating substances and is also less likely to promote insulin resistance, suggesting a potential physiological mechanism for lower blood pressure (BP) [27-30].

Despite these studies, few studies have assessed BP individually $[18,19,25,31,32]$ and thus, it remains unclear whether leg adiposity is independently associated with $\mathrm{BP}$ and hypertension. It is also unknown whether favorable associations for leg adiposity can be generalized to overall appendicular adiposity (both upper and lower extremities). There is a lack of recent, nationally representative studies assessing the role of appendicular adiposity in hypertension according to newer American College of Cardiology/American Heart Association (ACC/AHA) guidelines, which have markedly increased those classified as hypertensive. Furthermore, to our knowledge there exist no studies on the association between appendicular adiposity and hypertension subtype, which is important to deconstruct as studies have demonstrated elevated BMI as a primary risk factor for IDH in young adults $[9,18-20]$.

Hence, in this study, we investigate the association between various appendicular adiposity measures and BP, hypertension, and hypertension subtypes in young- to middle-aged US adults, with the purpose of better understanding the role of body fat distribution and its potential for early identification of hypertension and cardiovascular risk.

\section{Methods}

National health and nutrition examination survey dataset and linkage

The National Health and Nutrition Examination Survey (NHANES) is a nationally representative survey of the civilian, noninstitutionalized US population. The survey, implemented by the National Center for Health Statistics, follows a complex, stratified, multistage probability design. Surveys are conducted in 2-year cycles. We combined four publicly available cycles (2011-2012, 20132014, 2015-2016, and 2017-2018 cycles) to create a final NHANES 2011 to 2018 dataset for this secondary analysis. Further information about the dataset and the sample design can be found elsewhere [33].

\section{Study population}

Among the total 39,426 participants included in the NHANES 2011 to 2018 surveys, 11,222 were aged 20 to 59 and had dual-energy X-ray absorptiometry (DXA) scan data $(N=28,204$ excluded). Among these participants, a further 745 were excluded due to self-reported history of cardiovascular disease (stroke, myocardial infarction, coronary artery disease, and/or heart failure). An additional 1706 who were taking antihypertensive medications and 1360 with missing data on covariates were excluded, yielding the final sample size of 7411 (3723 male and 3688 non-pregnant female) (Fig. S1).

\section{Variables of interest}

Our primary predictor was the average percentage of whole-body fat mass present in the lower extremities (termed leg/total\%) and upper extremities (arm/total\%). Secondary predictors included (1) the average lean mass-to-fat mass ratio between each lower extremity (termed leg lean/fat ratio) or upper extremity (arm lean/ fat ratio), and (2) average percentage of extremity mass (excluding bone mineral content) that was fat (leg fat\%, arm fat\%). Trained and certified radiology technicians acquired and calculated these measurements through a whole-body scan using Hologic Discovery model A densitometers (Hologic Inc., Bedford, MA, USA). Further details about the DXA scan and quality control measures are available elsewhere [34]. Each predictor was also 
categorized into sex-specific tertiles-leg/total\% (female: $<36,36-41 \%$, $\geq 41 \%$; male: $<31.5,31.5-36.0 \%$, $\geq 36 \%$ ); leg lean/fat ratio (female: <1.1, 1.1-1.4, $\geq 1.4$; male: $<2.3$, $2.3-3.0$, $\geq 3.0$ ); leg fat $\%$ (female: $<40,40-45 \%$, $\geq 45 \%$; male: <24, 24-29\%, $\geq 29 \%$ ); arm/total \% (female: < 11.6, $11.6-12.9 \%$, $\geq 12.9 \%$; male: $<11.3,11.3-12.2 \%, \geq 12.2 \%$; arm lean/fat ratio (female: <1.1, 1.1-1.5, $\geq 1.5$; male: < $2.6,2.6-3.5, \geq 3.5$ ); arm fat \% (female: $<39,39-45 \%$, $\geq 45 \%$ male: $<21,21-26 \%, \geq 26 \%$ ).

Our primary outcomes included hypertension and hypertension subtype. Hypertension was diagnosed as per the 2017 ACC/AHA guidelines $(\geq 130 / 80 \mathrm{mmHg})$ [35] and hypertension subtypes were diagnosed as follows: (1) ISH: systolic BP (SBP) $\geq 130$ and diastolic BP (DBP) < 80; (2) IDH: SBP < 130 and DBP $\geq 80$; (3) SDH: $\mathrm{SBP} \geq 130$ and $\mathrm{DBP} \geq 80$; (4) normotension: $\mathrm{SBP}<130$ and $\mathrm{DBP}<80$.

Secondary outcomes included SBP, DBP, and pulse pressure, treated as continuous variables. Auscultatory, sitting BP measurements were performed using a mercury sphygmomanometer (Baumanometer ${ }^{\circ}$ - Gravity $\mathrm{Rx}$ model; W.A. Baum Co., Inc., Copiague, NY, USA). All staff followed strict quality control and protocol-driven procedures that are available elsewhere [36]. BP was measured three times consecutively by a trained physician during a mobile examination center visit. Participants were asked to sit and rest for $5 \mathrm{~min}$ before the first measurement. There was a thirty-second interval between measurements. We took the average of the three BP measurements.

Our covariates included demographic factors (age, race/ethnicity, poverty-income ratio [median household income/poverty threshold]), sociobehavioral factors (education level, physical activity, alcohol consumption, smoking status as determined by serum cotinine levels), and cardiometabolic variables (BMI, waist circumference, high density lipoprotein [HDL], triglycerides, Creactive protein, diabetes status [hemoglobin A1c, HbA1c $>6.5 \%$, fasting glucose $\geq 126 \mathrm{mg} / \mathrm{dL}$, self-reported diagnosis of diabetes, oral or insulin medication use], serum creatinine, and alanine aminotransferase). Measurement protocols for each covariate are described elsewhere [36].

\section{Statistical analysis}

As an initial, exploratory analysis, we first plotted a $20 \%$ representative subsample of the data and determined the correlation between BP and leg adiposity measures, stratified by sex. We then used multivariable linear regression, assuming normality, to estimate mean changes in BP overall and stratified by BMI group. As sex did not significantly modify the effect of leg adiposity on BP ( $\mathrm{P}$ for interaction $=0.25$ ), subsequent analyses used sexspecific categories, but did not stratify by sex.
To compare baseline characteristics across leg/total\% tertiles, we used one-way ANOVA for continuous variables and Rao-Scott chi-square tests for categorical variables. We also compared baseline characteristics across hypertension status in both males and females and used identical statistical analyses. To determine the independent association between appendicular adiposity and overall hypertension, we used multivariable logistic regression, adjusting for covariates in a series of three additive models. Model 1 adjusted for demographic factors (age, sex, race/ethnicity, poverty index). Model 2 adjusted for Model 1 factors + sociobehavioral and cardiometabolic factors (SBP, heart rate, triglycerides, BMI, truncal fat mass, HDL, alanine aminotransferase, diabetes status, serum creatinine, albuminuria, smoking, alcohol consumption). Model 3 adjusts for other examination factors (leg fat parameters other than main predictor, arm fat parameters other than main predictor). There was no significant multicollinearity between covariates as determined by variance inflation factor. To determine the independent association between predictors and hypertension subtypes, we estimated relative risk ratios (RRRs) using multinomial logistic regression in a series of three additive models as described for the logistic regression. Finally, we determined the predictive value of our adiposity measures using the c-statistic, an estimate of the area-under-receiver operator curve (AUC).

Analysis was stratified by BMI group (low/normal, overweight, and obese) to account for differences in fat distribution and content. All analyses utilized weights to produce nationally representative estimates and account for the oversampling of minorities (e.g., elderly, African Americans, and Mexican Americans). Specifically, we utilized NHANES examination sample weights for each cycle and divided by 4 to obtain pooled weights across four survey cycles. We did not adjust for statistical significance for multiple comparisons. All analyses also accounted for the complex survey design with strata and cluster variables using a traditional Taylor linearization approach to obtain accurate variance estimates using SAS survey procedures. All analyses followed NHANES analytic guidelines [33]. Analyses were conducted using SAS ver. 9.4 (SAS Institute Inc., Cary, NC, USA) with a significance level of 0.05 .

\section{Results}

Among the 7411 participants, the average age (standard deviation, SD) of the 3723 males and 3688 females was $36.9(0.3)$ and $37.8(0.3)$ years, respectively. Males who had hypertensive BPs $(1158,29.7 \%)$ were older, had higher BMI, truncal fat, and waist circumference, higher triglyceride levels, and higher $\mathrm{HbA} 1 \mathrm{c} \%$. On the contrary, hypertensive males had lower leg/total\% and leg and 
arm lean/fat ratio, but no difference in arm/total\%, HDL, and low-density lipoprotein (LDL) compared to normotensive males (Table S1). Females followed similar patterns in baseline characteristics (Table S2).

When comparing baseline characteristics by sexspecific tertiles of leg/total\%, those in the highest tertile (T3) of leg/total\% were younger, less educated, and of lower BMI and waist circumference. They also had lower leg lean/fat ratio, larger average leg area and upper leg length, higher arm lean/fat ratio, and no significant difference in average arm area. BP was significantly lower with increasing leg/total\% tertile. Further, those in T3 had a more favorable metabolic and lipid panel, with a $38.2 \%$ difference in prevalence of hypertriglyceridemia and $26 \%$ difference in impaired glucose tolerance between the lowest and highest tertiles (Table 1).

Scatterplots of leg adiposity measures with SBP and DBP showed significant negative correlations for leg lean/fat ratio and leg/total\%, but a positive correlation for leg fat\%. Leg/total\% showed the greatest magnitude of correlation, at $r=0.20$ in males and $r=0.25$ in females (Fig. S2). Sex did not significantly modify the association between leg adiposity and BP (P for interaction $=0.25$ ).

Nearly $24 \%$ of participants had hypertension. Those in T3 of leg/total\% had 30\% lower adjusted ORs (aORs) of hypertension compared to the lowest tertile ( $\mathrm{T} 1$; aOR, 0.70; 95\% confidence interval [CI], 0.55-0.89) (Table 2). Those in tertile 2 also displayed significantly reduced odds of hypertension compared to $\mathrm{T} 1$ (aOR, 0.81; 95\% CI, 0.66-0.99). These associations did not stay significant for leg lean/fat ratio and leg fat\% tertiles, losing their significance in Model 2, after adjustment for cardiometabolic factors, most importantly SBP. The corresponding tertiles for arm measures did not yield significant associations with hypertension (Table 3).

Stratifying by BMI group revealed attenuated, lower odds of hypertension for leg/total\% with increasing BMI, although differences between BMI groups remained insignificant $(\mathrm{P}$ for interaction $=0.40)$ (Table 4$)$. Among the low/normal BMI group, those in T3 of leg/total\% had $46 \%$ lower adjusted odds of hypertension compared to T1. Among overweight individuals and obese individuals, there was no significant difference in odds of hypertension between T3 and T1 (Table 4). Overweight individuals in $\mathrm{T} 3$ of leg lean/fat ratio and arm lean/fat ratio had 73 and $102 \%$, respectively, higher odds of hypertension compared to $\mathrm{T} 1$.

To investigate the association between leg/total\% and hypertension, we divided hypertension into its subtypes and determined the RRR of each hypertension subtype relative to normotension (Table 5, Fig. S3). Those in T3 of leg/total\% had $49 \%$ decreased odds of IDH, $41 \%$ decreased odds of SDH, but no significant change in odds of ISH. Arm adiposity measures followed similar patterns, although were of lesser significance. Unique to arm adiposity measures was a significant $46 \%$ decrease in the relative odds of ISH for T3 of arm fat\% as well as a $111 \%$ increase in the relative odds of ISH for T3 of arm lean/fat ratio compared to T1 (Table 5).

As an exploratory analysis, we treated appendicular adiposity and BP as continuous and did identical analyses stratified by sex (Tables 6 , S3-S9). For every $1 \%$ increase in leg/total\%, DBP decreased by an adjusted mean $0.35 \mathrm{mmHg}(95 \% \mathrm{CI},-0.48$ to -0.22$)$ in males and 0.18 $\mathrm{mmHg}(95 \% \mathrm{CI},-0.28$ to -0.08$)$ in females (Table 6). This association did not hold for SBP in males. Leg lean/ fat ratio was positively associated with SBP, while leg fat $\%$ was negatively associated with SBP but not DBP in both males and females ( $\beta[95 \% \mathrm{CI}]$; male: lean/fat ratio, 1.11 [0.34 to 1.89 ]; leg fat\%, -0.22 [-0.34 to -0.09$]$; female: lean/fat ratio, 2.15 [0.66 to 3.65]); leg fat\%, -0.16 [-0.26 to -0.06$])$. Arm adiposity measures demonstrated similar associations, with the exception that in males, arm fat\% was inversely associated with DBP as well as SBP (Table S5). By comparison, every $1 \%$ increase in truncal fat results in an average DBP increase of $0.084 \mathrm{mmHg}$ ( $95 \% \mathrm{CI}, 0.003$ to 0.165 ) and an SBP decrease of $0.148 \mathrm{mmHg}$ ( $95 \% \mathrm{CI}, 0.06$ to 0.235 ).

When stratifying by BMI category, we found that BMI only modestly, insignificantly modified the effect of appendicular adiposity on BP (Tables S6, 7). In males, while leg/total\% displayed a significant, inverse association with DBP across all BMI categories, the associations for leg lean/fat ratio and leg fat\% were only significant in overweight and obese males (Table S6). In females, BMI stratification nullified the associations between appendicular adiposity and BP; only arm lean/fat ratio remained positively associated with SBP across all BMI categories (Table S7).

When we assessed continuous BP changes across tertiles of adiposity, males and females in the highest tertile (T3) of leg/total\% had decreased mean DBP and those in T3 of leg lean/fat ratio had increased mean SBP, similar to findings treating adiposity as continuous (Table S3). As differences between males and females were not significant, initial analyses done combining sexes but still accounting for sex-specific tertiles were justified.

The predictive value of leg/total\% was determined by estimating the AUC using the c-statistic. We found that leg/total\% had a c-statistic of 0.644 , whereas arm/total\% had a c-statistic of 0.509 . Leg/total\% had similar, but slightly lower, c-statistic than both age and waist circumference and higher c-statistic than BMI (Table S10).

\section{Discussion}

In this nationally representative study of 7411 young- to middle-aged adults, we found that leg adiposity, as measured via leg/total\% and leg fat\%, as well as 
Table 1 Baseline characteristics by leg adiposity (leg/total\%) tertile

\begin{tabular}{|c|c|c|c|c|}
\hline Variable & Tertile $1^{a}$ & Tertile $2^{a}$ & Tertile $3^{a}$ & $P$-value ${ }^{\mathrm{b}}$ \\
\hline \multicolumn{5}{|l|}{ Demographics } \\
\hline Age (yr) & $41.6(0.3)$ & $37.5(0.3)$ & $33.7(0.4)$ & $<0.0001$ \\
\hline \multicolumn{5}{|l|}{ Race/ethnicity } \\
\hline Mexican American & $672(19.6 \%)$ & $357(10.9 \%)$ & $171(5.0 \%)$ & \multirow[t]{6}{*}{$<0.0001$} \\
\hline Other Hispanic & $295(8.7 \%)$ & $274(7.8 \%)$ & $219(6.3 \%)$ & \\
\hline Non-Hispanic White & $734(56.5 \%)$ & 857 (61.3\%) & $1037(66.6 \%)$ & \\
\hline Non-Hispanic Black & 199 (4.6\%) & $395(8.5 \%)$ & 735 (14.4\%) & \\
\hline Non-Hispanic Asian & $417(7.2 \%)$ & $450(7.5 \%)$ & $301(4.6 \%)$ & \\
\hline Other race & 87 (3.3\%) & 119 (4.0\%) & $92(3.1 \%)$ & \\
\hline Poverty-income ratio & $2.9(0.06)$ & $3.0(0.06)$ & $3.0(0.08)$ & 0.1095 \\
\hline \multicolumn{5}{|l|}{ Education level } \\
\hline$<$ High school & 1300 (62.1\%) & $1552(66.9 \%)$ & $1706(71.8 \%)$ & \multirow[t]{3}{*}{$<0.0001$} \\
\hline High school & $514(21.2 \%)$ & 519 (21.3\%) & $540(19.5 \%)$ & \\
\hline > High school & $590(16.7 \%)$ & $381(11.8 \%)$ & 309 (8.7\%) & \\
\hline \multicolumn{5}{|l|}{ Sociobehavioral factors } \\
\hline \multicolumn{5}{|l|}{ Smoking status ${ }^{c}$} \\
\hline Low/none & $829(37.4 \%)$ & $746(33.6 \%)$ & $672(32.4 \%)$ & \multirow[t]{3}{*}{0.0070} \\
\hline Moderate & $972(36.4 \%)$ & $1047(40.5 \%)$ & 1032 (37.7\%) & \\
\hline High & $603(26.2 \%)$ & $659(26.0 \%)$ & 847 (29.9\%) & \\
\hline Alcohol consumption (drinks/day) & $3.3(0.3)$ & $3.2(0.3)$ & $3.1(0.2)$ & 0.7734 \\
\hline \multicolumn{5}{|l|}{ Examination measurements } \\
\hline BMI $\left(\mathrm{kg} / \mathrm{m}^{2}\right)$ & $30.6(0.2)$ & $28.0(0.2)$ & $25.5(0.2)$ & $<0.0001$ \\
\hline Waist circumference (cm) & $103.4(0.4)$ & $95.8(0.4)$ & $87.7(0.4)$ & $<0.0001$ \\
\hline Truncal fat percent (\%) & $34.9(0.2)$ & $31.6(0.2)$ & $26.9(0.2)$ & $<0.0001$ \\
\hline$\%$ Total fat in lower extremities ${ }^{d}$ & $30.3(0.1)$ & $36.1(0.1)$ & $42.2(0.1)$ & $<0.0001$ \\
\hline Leg lean mass/fat mass ratio ${ }^{d}$ & $2.1(0.03)$ & $2.0(0.03)$ & $1.9(0.02)$ & $<0.0001$ \\
\hline$\%$ Fat in total leg mass ${ }^{d}$ & $33.4(0.3)$ & $35.3(0.3)$ & $35.9(0.3)$ & $<0.0001$ \\
\hline Average leg area $\left(\mathrm{cm}^{2}\right)$ & $365.8(1.4)$ & $371.7(1.2)$ & $376.9(1.3)$ & $<0.0001$ \\
\hline Upper leg length (cm) & $38.7(0.1)$ & $39.9(0.1)$ & $40.6(0.1)$ & $<0.0001$ \\
\hline$\%$ Total fat in upper extremities & $12.4(0.04)$ & $12.0(0.04)$ & $11.7(0.04)$ & $<0.0001$ \\
\hline Arm lean mass/fat mass ratio & $2.1(0.03)$ & $2.3(0.03)$ & $2.5(0.03)$ & $<0.0001$ \\
\hline$\%$ Fat in total arm mass & $35.0(0.3)$ & $33.3(0.3)$ & $30.8(0.3)$ & $<0.0001$ \\
\hline Average arm area $\left(\mathrm{cm}^{2}\right)$ & $233.0(1.1)$ & $232.2(0.9)$ & $231.4(0.9)$ & 0.5127 \\
\hline Heart rate (bpm) & $73.7(0.4)$ & $73.1(0.3)$ & $71.6(0.3)$ & $<0.0001$ \\
\hline $\mathrm{SBP}(\mathrm{mmHg})$ & $120.3(0.4)$ & $116.0(0.3)$ & $113.7(0.3)$ & $<0.0001$ \\
\hline $\mathrm{DBP}(\mathrm{mmHg})$ & $73.7(0.3)$ & $70.7(0.3)$ & $68.1(0.3)$ & $<0.0001$ \\
\hline Pulse pressure (mmHg) & $46.6(0.4)$ & $45.3(0.4)$ & $45.6(0.4)$ & 0.031 \\
\hline Average maximum SBP (mmHg) & $123.2(0.4)$ & $118.8(0.3)$ & $116.5(0.3)$ & $<0.0001$ \\
\hline Maximum - minimum SBP (mmHg) & $5.8(0.1)$ & $5.6(0.1)$ & $5.6(0.1)$ & 0.18 \\
\hline Maximum - minimum DBP $(\mathrm{mmHg})$ & $5.7(0.1)$ & $5.9(0.2)$ & $6.1(0.2)$ & 0.027 \\
\hline \multicolumn{5}{|l|}{ Laboratory measurements } \\
\hline Plasma glucose (mg/dL) & $109.2(0.9)$ & $97.6(0.6)$ & $94.8(0.5)$ & $<0.0001$ \\
\hline Hemoglobin A1c (\%) & $5.7(0.03)$ & $5.4(0.01)$ & $5.2(0.01)$ & $<0.0001$ \\
\hline Serum insulin (uU/mL) & $16.1(0.8)$ & $10.5(0.4)$ & $8.0(0.3)$ & $<0.0001$ \\
\hline
\end{tabular}


Table 1 Baseline characteristics by leg adiposity (leg/total\%) tertile (Continued)

\begin{tabular}{|c|c|c|c|c|}
\hline Variable & Tertile $1^{\mathrm{a}}$ & 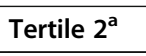 & Tertile $3^{a}$ & $P$-value ${ }^{\mathrm{b}}$ \\
\hline Serum HDL (mg/dL) & $47.7(0.4)$ & $53.6(0.5)$ & $58.8(0.5)$ & $<0.0001$ \\
\hline Serum LDL (mg/dL) & $123.6(2.5)$ & $116.3(1.8)$ & $106.0(1.7)$ & $<0.0001$ \\
\hline Serum triglycerides (mg/dL) & $197.0(4.0)$ & $134.3(2.5)$ & $98.6(1.4)$ & $<0.0001$ \\
\hline ALT (IU/L) & $29.9(0.5)$ & $24.9(0.5)$ & $20.9(0.3)$ & $<0.0001$ \\
\hline AST (IU/L) & $26.1(0.5)$ & $24.2(0.3)$ & $23.0(0.2)$ & $<0.0001$ \\
\hline Serum albumin (g/dL) & $4.29(0.01)$ & $4.33(0.01)$ & $4.39(0.01)$ & $<0.0001$ \\
\hline Serum creatinine & $0.83(0.01)$ & $0.85(0.01)$ & $0.85(0.01)$ & 0.0006 \\
\hline Serum creatine phosphokinase & $152.2(5.0)$ & $159.1(6.8)$ & $157.5(5.2)$ & 0.6855 \\
\hline \multicolumn{5}{|l|}{ Comorbidities } \\
\hline Impaired glucose tolerance ${ }^{e}$ & $1138(43.3 \%)$ & $751(24.6 \%)$ & $516(16.9 \%)$ & $<0.0001$ \\
\hline Hypertriglyceridemia & $1258(53.1 \%)$ & $753(30.5 \%)$ & $381(14.9 \%)$ & $<0.0001$ \\
\hline Anti-diabetes medication use & $148(4.5 \%)$ & $32(0.8 \%)$ & $10(0.3 \%)$ & $<0.0001$ \\
\hline Cholesterol medication use & $161(7.7 \%)$ & $74(3.6 \%)$ & $29(1.6 \%)$ & $<0.0001$ \\
\hline HOMA-IR & $474(54.5 \%)$ & $271(27.5 \%)$ & 144 (13.8\%) & $<0.0001$ \\
\hline
\end{tabular}

Continuous variables represented as weighted mean (SD). Categorical variables represented as unweighted $\mathrm{N}$ (weighted \%)

$S D$ standard deviation, $B M I$ body mass index, SBP systolic blood pressure, $D B P$ diastolic blood pressure, HDL high density lipoprotein, $L D L$ low-density lipoprotein, ALT alanine aminotransferase, AST aspartate aminotransferase, HOMA-IR Homeostatic Model Assessment of Insulin Resistance

${ }^{a}$ Tertiles are of leg/total \% (percentage of total body fat mass that is in bilateral lower extremities). Sex-specific tertiles are as follows: female: $<36,36-41 \%, \geq 41 \%$; male: $<31.5,31.5-36.0 \%, \geq 36 \%$

${ }^{\mathrm{b}} P$-value calculated using one-way ANOVA for continuous variables and Rao-Scott chi-square tests for categorical variables. $P$-values and/or significance levels were not adjusted for multiple comparisons as this table was considered exploratory in nature

'Smoking categories determined by serum cotinine levels

${ }_{d} \%$ Total fat in lower extremities corresponds to leg/total \%, leg lean mass/fat mass ratio corresponds to leg lean/fat ratio, \% fat in total leg mass corresponds to leg fat \%. Identical for arm measures

eImpaired glucose tolerance was defined as hemoglobin A1c $\geq 5.7 \%$, fasting plasma glucose $\geq 100 \mathrm{mg} / \mathrm{dL}$, self-reported history of diabetes or self-reported history of oral anti-diabetes medications or insulin therapy

fData on HOMA-IR only available for 2756 individuals (614 with hypertension). HOMA = fasting glucose $(\mathrm{mg} / \mathrm{dL}) \times$ insulin $(\mathrm{uU} / \mathrm{mL}) / 405$. HOMA $\geq 3$ signifies insulin resistance

Table 2 Odds of hypertension by tertiles of leg adiposity measures

\begin{tabular}{lllll}
\hline Variable & & Tertile 1 & Tertile 2 & Tertile 3 \\
\hline No. (\%) & 1853 (23.8) & - & - & - \\
Leg/total \% & Unadjusted & 1.00 (Ref) & $0.57(0.47,0.69)$ & $0.35(0.29,0.42)$ \\
& Model 1 & 1.00 (Ref) & $0.63(0.52,0.78)$ & $0.44(0.36,0.54)$ \\
& Model 2 & 1.00 (Ref) & $0.79(0.65,0.97)$ & $0.68(0.56,0.82)$ \\
& Model 3 & 1.00 (Ref) & $0.81(0.66,0.99)$ & $0.70(0.55,0.89)$ \\
Leg lean/fat ratio & Unadjusted & 1.00 (Ref) & $0.83(0.69,0.98)$ & $0.69(0.57,0.83)$ \\
& Model 1 & 1.00 (Ref) & $0.86(0.73,1.02)$ & $0.74(0.61,0.90)$ \\
& Model 2 & 1.00 (Ref) & $1.19(0.99,1.43)$ & $1.37(1.07,1.74)$ \\
Leg fat \% & Model 3 & 1.00 (Ref) & $1.11(0.92,1.34)$ & $1.23(0.95,1.60)$ \\
& Unadjusted & 1.00 (Ref) & $1.20(0.97,1.45)$ & $1.44(1.19,1.73)$ \\
& Model 1 & 1.00 (Ref) & $0.90(0.74,1.11)$ & $1.38(1.14,1.70)$ \\
& Model 2 & 1.00 (Ref) & $0.93(0.75,1.15)$ & $0.75(0.59,0.95)$ \\
\hline
\end{tabular}

Data are presented as odds ratio (95\% confidence interval)

Model 1 adjusts for demographic factors (age, sex, race/ethnicity, poverty index). Model 2 adjusts for Model 1 factors + cardiometabolic factors (systolic blood pressure, heart rate, triglycerides, body mass index, truncal fat mass, high density lipoprotein, alanine aminotransferase, diabetes status, serum creatinine, albuminuria, smoking, alcohol consumption). Model 3 adjusts for other examination factors (leg fat parameters other than main predictor, arm fat parameters other than main predictor). Lean/fat ratio and fat \% variables were not included in the same model due to significant correlation and high variance inflation factor. \% Total fat in lower extremities corresponds to leg/total \%, leg lean mass/fat mass ratio corresponds to leg lean/fat ratio, \% fat in total leg mass corresponds to leg fat \%. Identical for arm measures. Tertiles are as follows: leg/total \% (female: $<36,36-41 \%, \geq 41 \%$; male: $<31.5,31.5-36.0 \%$, $\geq 36 \%$ ); leg lean/fat ratio (female: $<1.1,1.1-1.4, \geq 1.40$; male: $<2.3,2.3-3.0, \geq 3.0$ ); leg fat $\%$ (female: $<40,40-45 \%, \geq 45 \%$; male: $<24,24-29 \%, \geq 29 \%$ ) 
Table 3 Odds of hypertension by tertiles of arm adiposity measures

\begin{tabular}{|c|c|c|c|c|}
\hline Variable & & Tertile 1 & Tertile 2 & Tertile 3 \\
\hline No. (\%) & $1853(23.8)$ & - & - & - \\
\hline \multirow[t]{4}{*}{ Arm/total \% } & Unadjusted & 1.00 (Ref) & $0.97(0.79,1.18)$ & $1.12(0.92,1.36)$ \\
\hline & Model 1 & 1.00 (Ref) & $0.99(0.72,1.35)$ & $1.24(0.87,1.75)$ \\
\hline & Model 2 & 1.00 (Ref) & $0.96(0.70,1.31)$ & $0.90(0.64,1.24)$ \\
\hline & Model 3 & 1.00 (Ref) & $0.87(0.62,1.22)$ & $0.89(0.68,1.17)$ \\
\hline \multirow[t]{4}{*}{ Arm lean/fat ratio } & Unadjusted & 1.00 (Ref) & $0.74(0.64,0.87)$ & $0.50(0.42,0.60)$ \\
\hline & Model 1 & 1.00 (Ref) & $0.77(0.42,1.40)$ & $0.63(0.36,1.11)$ \\
\hline & Model 2 & 1.00 (Ref) & $0.99(0.73,1.33)$ & $1.17(0.81,1.70)$ \\
\hline & Model 3 & 1.00 (Ref) & $1.03(0.81,1.31)$ & $1.13(0.71,1.80)$ \\
\hline \multirow[t]{4}{*}{ Arm fat $\%$} & Unadjusted & 1.00 (Ref) & $1.49(1.26,1.77)$ & $2.03(1.72,2.40)$ \\
\hline & Model 1 & 1.00 (Ref) & $1.26(0.95,1.69)$ & $1.66(1.02,2.69)$ \\
\hline & Model 2 & 1.00 (Ref) & $0.84(0.65,1.10)$ & $0.92(0.67,1.25)$ \\
\hline & Model 3 & 1.00 (Ref) & $1.07(0.63,1.80)$ & $0.97(0.58,1.61)$ \\
\hline
\end{tabular}

Data are presented as odds ratio (95\% confidence interval)

Model 1 adjusts for demographic factors (age, sex, race/ethnicity, poverty index). Model 2 adjusts for Model 1 factors + cardiometabolic factors (systolic blood pressure, heart rate, triglycerides, body mass index, truncal fat mass, high density lipoprotein, alanine aminotransferase, diabetes status, serum creatinine, albuminuria, smoking, alcohol consumption). Model 3 adjusts for other examination factors (leg fat parameters other than main predictor, arm fat parameters other than main predictor)

$\%$ Total fat in upper extremities corresponds to arm/total \%, arm lean mass/fat mass ratio corresponds to arm lean/fat ratio, \% fat in total arm mass corresponds to arm fat \%. Tertiles are as follows: arm/total \% (female: $<11.6,11.6-12.9 \%, \geq 12.9 \%$; male: $<11.3,11.3-12.2 \%$, $\geq 12.2 \%$ ); arm lean/fat ratio (female: $<1.1,1.1-1.5$, $\geq 1.5$; male: $<2.6,2.6-3.5, \geq 3.5$ ); arm fat $\%$ (female: $<39,39-45 \%, \geq 45 \%$; male: $<21,21-26 \%, \geq 26 \%$ )

Table 4 Association between appendicular adiposity and HTN by BMI group

\begin{tabular}{lllll}
\hline Variable & & BMI group & & \\
\cline { 4 - 5 } & & Low/normal & Overweight & Obese \\
\hline No. $(\%$ HTN) & & $443(16.9)$ & $612(31.7)$ & $798(52.1)$ \\
Leg/total \% & Unadjusted & $0.30(0.21,0.43)$ & $0.40(0.28,0.58)$ & $0.79(0.58,1.07)$ \\
& Fully adjusted ${ }^{a}$ & $0.53(0.30,0.96)$ & $0.91(0.58,1.43)$ & $0.92(0.63,1.33)$ \\
Arm/total \% & Unadjusted & $1.24(0.87,1.75)$ & $0.90(0.64,1.24)$ & $0.89(0.68,1.17)$ \\
& Fully adjusted & $1.00(0.69,1.45)$ & $0.87(0.62,1.23)$ & $0.85(0.62,1.16)$ \\
Leg lean/fat ratio & Unadjusted & $1.01(0.63,1.61)$ & $1.45(1.04,2.02)$ & $1.05(0.72,1.53)$ \\
& Fully adjusted & $1.00(0.54,1.84)$ & $1.73(1.16,2.56)$ & $1.20(0.75,1.91)$ \\
Arm lean/fat ratio & Unadjusted & $0.63(0.36,1.11)$ & $1.17(0.81,1.70)$ & $1.13(0.71,1.80)$ \\
Leg fat\% & Fully adjusted & $0.92(0.48,1.77)$ & $2.02(1.29,3.17)$ & $1.43(0.93,2.20)$ \\
Arm fat\% & Unadjusted & $1.15(0.78,1.69)$ & $0.61(0.44,0.86)$ & $1.02(0.69,1.49)$ \\
& Fully adjusted & $1.20(0.63,2.29)$ & $0.63(0.38,1.03)$ & $1.15(0.66,1.98)$ \\
\hline
\end{tabular}

Data are presented as odds ratio ( $95 \%$ confidence interval). Odds ratio represent highest tertile vs. lowest tertile for each adiposity measure

HTN hypertension, $B M I$ body mass index, $O R$ odds ratio

${ }^{a}$ Fully adjusted model is identical to Model 3. Model 3 adjusts for other examination factors (leg fat parameters other than main predictor, arm fat parameters other than main predictor). Lean/fat ratio and fat $\%$ variables were not included in the same model due to significant correlation and high variance inflation factor $\%$ Total fat in lower extremities corresponds to leg/total \%, leg lean mass/fat mass ratio corresponds to leg lean/fat ratio, \% fat in total leg mass corresponds to leg fat \%. Identical for arm measures. Tertiles are as follows: leg/total \% (female: $<36,36-41 \%, \geq 41 \%$; male: $<31.5,31.5-36.0 \%, \geq 36 \%$ ); leg lean/fat ratio (female: < $1.10,1.10-1.40, \geq 1.40$; male: $<2.30,2.30-3.00, \geq 3.00$ ); leg fat $\%$ (female: $<40,40-45 \%, \geq 45 \%$; male: $<24,24-29 \%, \geq 29 \%$ ). Arm measures: arm/total \% (female: $<$ 11.6, 11.6-12.9\%, $\geq 12.9 \%$; male: $<11.3,11.3-12.2 \%, \geq 12.2 \%$ ); arm lean/fat ratio (female: $<1.1,1.1-1.5, \geq 1.5$; male: $<2.6,2.6-3.5, \geq 3.5$ ); arm fat $\%$ (female: $<39,39-$ $45 \%$, $\geq 45 \%$; male: $<21,21-26 \%, \geq 26 \%$ ) 
Table 5 Association between leg adiposity and hypertension subtypes

\begin{tabular}{|c|c|c|c|c|}
\hline Variable & & Tertile 1 & Tertile 2 & Tertile 3 \\
\hline \multirow[t]{4}{*}{ Leg/total\% } & Normotension & 1.00 (Ref) & & \\
\hline & $\mathrm{IDH}$ & 1.00 (Ref) & $0.79(0.59,1.06)$ & $0.51(0.37,0.70)$ \\
\hline & $\mathrm{SDH}$ & 1.00 (Ref) & $0.70(0.50,0.98)$ & $0.59(0.43,0.80)$ \\
\hline & ISH & 1.00 (Ref) & $0.84(0.61,1.16)$ & $1.06(0.70,1.59)$ \\
\hline \multirow[t]{4}{*}{ Leg lean/fat ratio } & Normotension & 1.00 (Ref) & & \\
\hline & $\mathrm{IDH}$ & 1.00 (Ref) & $0.99(0.73,1.34)$ & $0.93(0.62,1.38)$ \\
\hline & $\mathrm{SDH}$ & 1.00 (Ref) & $1.26(0.95,1.67)$ & $1.50(0.97,2.32)$ \\
\hline & ISH & 1.00 (Ref) & $1.16(0.83,1.63)$ & $1.56(1.00,2.41)$ \\
\hline \multirow[t]{4}{*}{ Leg fat $\%$} & Normotension & 1.00 (Ref) & & \\
\hline & $\mathrm{IDH}$ & 1.00 (Ref) & $1.17(0.89,1.54)$ & $1.04(0.72,1.52)$ \\
\hline & $\mathrm{SDH}$ & 1.00 (Ref) & $0.79(0.55,1.13)$ & $0.67(0.44,1.04)$ \\
\hline & ISH & 1.00 (Ref) & $0.76(0.54,1.08)$ & $0.69(0.43,1.10)$ \\
\hline \multirow[t]{4}{*}{ Arm/total\% } & Normotension & 1.00 (Ref) & & \\
\hline & $\mathrm{IDH}$ & 1.00 (Ref) & $0.99(0.73,1.34)$ & $0.70(0.52,0.95)$ \\
\hline & $\mathrm{SDH}$ & 1.00 (Ref) & $0.85(0.62,1.16)$ & $0.75(0.55,1.02)$ \\
\hline & ISH & 1.00 (Ref) & $1.16(0.82,1.64)$ & $1.21(0.88,1.65)$ \\
\hline \multirow[t]{4}{*}{ Arm lean/fat ratio } & Normotension & 1.00 (Ref) & & \\
\hline & $\mathrm{IDH}$ & 1.00 (Ref) & $1.11(0.82,1.50)$ & $1.12(0.76,1.65)$ \\
\hline & $\mathrm{SDH}$ & 1.00 (Ref) & $1.13(0.82,1.54)$ & $1.30(0.84,2.01)$ \\
\hline & ISH & 1.00 (Ref) & $1.28(0.94,1.75)$ & $2.11(1.28,3.46)$ \\
\hline \multirow[t]{4}{*}{ Arm fat $\%$} & Normotension & 1.00 (Ref) & & \\
\hline & $\mathrm{IDH}$ & 1.00 (Ref) & $0.94(0.71,1.25)$ & $0.96(0.66,1.41)$ \\
\hline & $\mathrm{SDH}$ & 1.00 (Ref) & $0.93(0.64,1.33)$ & $0.72(0.46,1.14)$ \\
\hline & ISH & 1.00 (Ref) & $0.64(0.45,0.92)$ & $0.54(0.34,0.86)$ \\
\hline
\end{tabular}

Data are presented as relative risk ratio (95\% confidence interval)

IDH isolated diastolic hypertension, $S D H$ systolic-diastolic hypertension, ISH isolated systolic hypertension

All estimates shown are fully adjusted via Model 3. Model 3 adjusts for other examination factors (leg fat parameters other than main predictor, arm fat

parameters other than main predictor). Lean/fat ratio and fat \% variables were not included in the same model due to significant correlation and high variance inflation factor

$\%$ Total fat in lower extremities corresponds to leg/total \%, leg lean mass/fat mass ratio corresponds to leg lean/fat ratio, \% fat in total leg mass corresponds to leg fat \%. Identical for arm measures. Tertiles are as follows: leg/total \% (female: $<36,36-41 \%, \geq 41 \%$; male: $<31.5,31.5-36.0 \%$, $\geq 36 \%$ ); leg lean/fat ratio (female: < $1.10,1.10-1.40, \geq 1.40$; male: $<2.30,2.30-3.00, \geq 3.00$ ); leg fat $\%$ (female: $<40,40-45 \%, \geq 45 \%$; male: $<24,24-29 \%, \geq 29 \%$ ). \% Total fat in upper extremities corresponds to arm/total \%, arm lean mass/fat mass ratio corresponds to arm lean/fat ratio, \% fat in total arm mass corresponds to arm fat \%. Tertiles are as follows: arm/total \% (female: $<11.6,11.6-12.9 \%, \geq 12.9 \%$; male: $<11.3,11.3-12.2 \%, \geq 12.2 \%$ ); arm lean/fat ratio (female: $<1.1,1.1-1.5, \geq 1.5 ;$ male: $<2.6,2.6-3.5$, $\geq 3.5$ ); arm fat $\%$ (female: $<39,39-45 \%, \geq 45 \%$; male: $<21,21-26 \%, \geq 26 \%$ )

corresponding arm adiposity measures were inversely associated with DBP and SBP, respectively. Arm and leg lean/fat ratio, on the other hand, were positively associated with SBP. Among the three measures of leg adiposity, only leg/total\% demonstrated a significant, $30 \%$ decreased odds of hypertension when comparing T3 ( $\geq 36 \%$ for male, $\geq 41 \%$ for female) to $\mathrm{T} 1 \quad(<31.5 \%$ for male, $<36 \%$ for female). Furthermore, leg/total\% decreased the odds of IDH and SDH, but not ISH relative to normotension. BMI modified the association between leg/total\% and hypertension, with low/normal BMI individuals showing significantly more protective odds than overweight and obese individuals. Interestingly, arm fat $\%$, not arm/total\%, was inversely associated with SBP and overall hypertension. Consequently, leg/total\% had a higher predictive value than arm/total\% but was insignificantly lower than waist circumference and age in predicting hypertension.

Our findings are in line with existing literature on the association between leg adiposity and various cardiometabolic conditions [13-32]. While prior studies have generally not focused on BP or hypertension as a primary outcome, they have consistently reported inverse associations between leg adiposity measures and metabolic risk factors. Zhang et al. [19], in a study of NHANES 1999 to 2006 participants with DXA scans, found that, regardless of ethnicity, leg adiposity indices (leg/total\%, leg fat\%, leg fat/truncal fat ratio) were all inversely associated with metabolic syndrome. Leg/total\%, however, had the strongest association. Other studies have 
Table 6 Association between leg adiposity and blood pressure

\begin{tabular}{|c|c|c|c|c|c|c|c|c|}
\hline \multirow[t]{2}{*}{ Variable } & & & \multicolumn{2}{|l|}{ SBP } & \multicolumn{2}{|l|}{ DBP } & \multicolumn{2}{|l|}{ Pulse Pressure } \\
\hline & & & $\beta(95 \% \mathrm{Cl})$ & $P$-value & $\beta(95 \% \mathrm{Cl})$ & $P$-value & $\beta(95 \% \mathrm{Cl})$ & $P$-value \\
\hline No. (\%) & $1853(23.8)$ & & - & - & - & - & - & - \\
\hline \multirow[t]{4}{*}{ Leg/total\% } & Male & Unadjusted & $-0.48(-0.58,-0.39)$ & $<0.0001$ & $-0.61(-0.70,-0.53)$ & $<0.0001$ & $0.13(0.021,0.24)$ & 0.0199 \\
\hline & & Fully adjusted & $-0.13(-0.29,0.022)$ & 0.093 & $-0.35(-0.48,-0.22)$ & $<0.0001$ & $0.21(0.042,0.37)$ & 0.015 \\
\hline & Female & Unadjusted & $-0.53(-0.62,-0.44)$ & $<0.0001$ & $-0.31(-0.36,-0.24)$ & $<0.0001$ & $-0.23(-0.31,-0.15)$ & $<0.0001$ \\
\hline & & Fully adjusted & $-0.17(-0.31,-0.034)$ & 0.015 & $-0.18(-0.28,-0.08)$ & 0.0007 & $-0.01(-0.15,0.14)$ & 0.94 \\
\hline \multirow[t]{4}{*}{ Leg lean/fat ratio } & Male & Unadjusted & $-0.84(-1.39,-0.29)$ & 0.0035 & $-1.29(-1.73,-0.86)$ & $<0.0001$ & $0.46(-0.11,1.03)$ & 0.11 \\
\hline & & Fully adjusted & $1.11(0.34,1.89)$ & 0.0058 & $-0.42(-1.24,0.40)$ & 0.31 & $1.53(0.54,2.52)$ & 0.0029 \\
\hline & Female & Unadjusted & $-2.67(-3.87,-1.47)$ & $<0.0001$ & $-1.72(-2.76,-0.69)$ & 0.0015 & $-0.95(-2.31,0.41)$ & 0.17 \\
\hline & & Fully adjusted & $2.15(0.66,3.65)$ & 0.0054 & $0.18(-1.26,1.62)$ & 0.80 & $1.97(0.25,3.70)$ & 0.025 \\
\hline \multirow[t]{4}{*}{ Leg fat $\%$} & Male & Unadjusted & $0.14(0.05,0.22)$ & 0.0026 & $0.17(0.11,0.23)$ & $<0.0001$ & $-0.032(-0.11,0.047)$ & 0.43 \\
\hline & & Fully adjusted & $-0.22(-0.34,-0.088)$ & 0.0013 & $0.046(-0.084,0.18)$ & 0.48 & $-0.26(-0.42,-0.11)$ & 0.0011 \\
\hline & Female & Unadjusted & $0.17(0.10,0.25)$ & $<0.0001$ & $0.10(0.036,0.16)$ & 0.0026 & $0.074(-0.0094,0.16)$ & 0.081 \\
\hline & & Fully adjusted & $-0.16(-0.26,-0.059)$ & 0.0024 & $-0.032(-0.13,0.061)$ & 0.49 & $-0.13(-0.25,-0.01)$ & 0.036 \\
\hline
\end{tabular}

SBP systolic blood pressure, DBP diastolic blood pressure, $\beta$ regression parameter estimate, $C l$ confidence interval (all estimates are of the fully adjusted model) Fully adjusted model is identical to Model 3 and adjusts for demographic factors (age, sex, race/ethnicity, poverty index), cardiometabolic factors (SBP, heart rate, triglycerides, body mass index, truncal fat mass, high density lipoprotein, alanine aminotransferase, diabetes status, serum creatinine, albuminuria, smoking, alcohol consumption), and other examination factors (leg fat parameters other than main predictor, arm fat parameters other than main predictor). Lean/fat ratio and fat $\%$ variables were not included in the same model due to significant correlation and high variance inflation factor

similarly found inverse associations between leg/total\% and cardiovascular risk factors [18-20], cardiovascular disease risk and all-cause mortality [21-26]. In fact, Han et al. [25], using the Korean NHANES, showed that leg/ total\% displayed the strongest association with atherosclerotic cardiovascular disease risk. Interestingly, studies that additionally assessed arm adiposity have found no significant association, and in some cases positive associations, with metabolic risk factors [17, 20,32, 33]. Our findings also corroborate a positive association between $\mathrm{arm} / \mathrm{total} \%$ and SBP and hypertension that was later attenuated by adjustment for cardiometabolic risk factors.

There continues to be variability in data concerning sex differences. Hu et al. [20], among White and Black Americans, showed that females generally had more protective associations of leg adiposity with metabolic syndrome criteria than males, Sakai et al. [18] demonstrated no difference between males and females for SBP or DBP after adjusting for menopausal status. While we also found no significant difference between sexes, we observed more protective odds of hypertension in females than males.

Of the four studies that have investigated the association between appendicular adiposity and BP/hypertension $[18,19,25,31]$, only one was conducted in a US representative population, all used $140 / 90$ as the hypertension threshold, and none have commented on hypertension subtype. Nevertheless, our findings corroborate those of the four studies. Sakai et al. [18] found a significant, inverse association between leg fat mass and hypertension $(\geq 140 / 90)$ as well as a mean $0.22 \mathrm{mmHg}$ decrease in DBP and $0.16 \mathrm{mmHg}$ decrease in SBP per 1 $\mathrm{kg}$ increase in leg fat mass in 4256 Japanese men aged 20 to 79 years. The authors also found an increase in BP for every $1 \mathrm{~kg}$ increase in lean leg mass, a counterintuitive finding that we also observed [18]. Increased lean mass, we speculate, may be associated with increased serum testosterone, an androgen that has been inconclusively linked to increased BP and cardiovascular disease [37]. Zhang et al. [19] showed a significant inverse correlation between leg/total\%, but not leg fat/leg mass ratio, and SBP and DBP. Han et al. [25], in the Korean NHANES population, found that those in T1 of leg/ total\% had nearly 3.5 times the odds of hypertension ( $\geq 140 / 90)$ compared to T3, although models were not adjusted for truncal adiposity or arm adiposity measures. Finally, Yan et al. [31], in a Chinese population, similarly demonstrated that leg/total\% was primary negative risk factor for hypertension $(\geq 140 / 90)$.

They clarified that this association was stronger in the non-obese population, a finding that we also demonstrated. This would seem to suggest that, even in those with low/normal BMI, body distribution of fat is important. However, after adjusting for truncal fat and waist circumference, leg/total\% continues to be a negative predictor of hypertension in low/normal BMI individuals. This suggests that, in addition to body distribution of fat, there exist a physiological difference between lower extremity adiposity and truncal or upper extremity adiposity. Conventionally, we have treated truncal fat as the primary source of fat that drives of metabolic disease; however, these studies and ours lead to the speculation 
that measurement of leg fat is just as important, and its effects independent of, truncal fat.

Not only is leg/total\% inversely associated with hypertension, it is also preferentially inversely associated with DBP and associated hypertension subtypes. This suggests that leg adiposity may a play a role in regulation of DBP, which is determined in large part by peripheral vascular resistance in arteriolar vessels. Although the biological mechanism behind leg fat's protective role is unknown, several studies have speculated and found that (1) leg fat is located primarily in subcutaneous tissue, as opposed to visceral tissue; (2) adipocytes in the lower extremities had lower free fatty acid turnover and lower rates of lipolysis; and (3) this decreased free fatty acid turnover may lead to decreased fatty acid concentrations in the blood and downregulation of triglyceride production [18, 19, 38].

We hypothesize that, in addition to decreased sympathetic and renin-angiotensin-aldosterone axis activation associated with subcutaneous fat, this downregulation of triglyceride production, which is corroborated by inverse associations between leg/total\% and triglyceride levels in studies assessing metabolic syndrome criteria, leads to decreased endothelial cell damage in arterioles and maintenance of vessel elasticity and compliance. This is further supported by Lee et al. [24], which found that every SD increase in leg/total\% was associated with a $1.03 \mathrm{~m} / \mathrm{sec}$ decrease in brachial-ankle pulse wave velocity, a measure of arterial stiffness.

There are several strengths of this study. We used a set of recent, large, nationally representative surveys with gold standard measurements of fat and lean mass through DXA scans. We comprehensively accounted for covariates to further confirm the independent association of leg/total\% and BP. By having a younger study population, we also minimized unmeasured chronic disease effects and effects of menopause on women. We utilized the 2017 ACC/AHA guidelines for hypertension, which not only includes more individuals in its hypertension diagnosis but emphasizes the notion that leg/ total\% may be important in those who are seemingly healthy.

Despite these strengths, there are limitations as well. This is a cross-sectional study so causality cannot be determined. It may be that those who are hypertensive tend to accumulate fat elsewhere. Further, while it is generalizable to those in the US aged 20 to 59 years, these results may not be generalizable to older adults $\geq 60$ years who also have disproportionately higher hypertension prevalence. While DXA scans are the gold standard measurement for body fat distribution, it cannot distinguish between subcutaneous and intramuscular fat in the legs, limiting our interpretation of results. Furthermore, visceral fat is still more clinically relevant and more strongly associated with cardiovascular disease risk, posing the question as to how clinically useful our results may be. Also, although our results are statistically significant, they may or may not be clinically significant. Although the absolute changes in BP are miniscule, it is important to note these BP differences are on a population level and across 1\% increments of appendicular adiposity. There may be residual confounding that is unaccounted for in this analysis, especially due to limited information on medication use and comorbidities (e.g., polycystic ovarian syndrome in women, hormone therapy).

\section{Conclusions}

In conclusion, a greater proportional distribution of total body fat around the legs is inversely, independently associated with DBP, while a greater proportion of fat in one's leg mass is inversely associated with SBP. Prospective studies on body fat distribution and incident hypertension are needed to understand the predictive value of leg adiposity and associated clinical surrogates (e.g., thigh circumference). These measures may help clinicians risk stratify patients to help improve hypertension screening and management. Technological improvements allowing for body part-specific fat measurement may also be in the horizon over the next decade and allow for further testing and clinical applicability of these leg adiposity measures.

\section{Abbreviations}

ACC/AHA: American College of Cardiology/American Heart Association; ALT: Alanine aminotransferase; AST: Aspartate aminotransferase; AUC: Areaunder-receiver operator curve; BMI: Body mass index; BP: Blood pressure;

Cl: Confidence interval; DBP: Diastolic blood pressure; DXA: Dual-energy X-ray absorptiometry; HbA1c: Hemoglobin A1c; HDL: High density lipoprotein; HOMA-IR: Homeostatic Model Assessment of Insulin Resistance; HTN: Hypertension; IDH: Isolated diastolic hypertension; ISH: Isolated systolic hypertension; LDL: Low-density lipoprotein; NHANES: National Health and Nutrition Examination Survey; OR: Odds ratio; RRR: Relative risk ratio; SBP: Systolic blood pressure; SD: Standard deviation; SDH: Systolic-diastolic hypertension; SE: Standard error; $\beta$ : Regression parameter estimate

\section{Supplementary Information}

The online version contains supplementary material available at https://doi. org/10.1186/s40885-021-00190-2.

Additional file 1: Table S1. Baseline characteristics in females by hypertension status. Table S2. Baseline characteristics in males by hypertension status. Table S3. Differences in mean blood pressure by tertiles of leg adiposity measures. Table S4. Average appendicular adiposity by hypertension subtype. Table S5. Association between arm adiposity and blood pressure. Table $\mathbf{S 6}$. Association between appendicular adiposity and SBP by BMI group in males. Table S7. Association between appendicular adiposity and SBP by BMI group in females. Table S8. Association between continuous leg adiposity measures and hypertension subtypes. Table S9. Association between continuous arm adiposity measures and hypertension subtypes. Table S10. estimated AUC for traditional and novel risk factors. Fig. S1. Exclusion cascade for final study sample from National Health and Nutrition Examination Survey 2011-2018. Fig. S2. Correlation between 
leg adiposity measures and blood pressure. Fig. S3. Prevalence of hypertension subtypes by leg/total \% tertiles.

\section{Acknowledgments}

None.

\section{Authors' contributions}

AV contributed to study design, data analysis, management, and manuscript writing. DL contributed to manuscript writing, data visualization. PM and BD contributed to data management, manuscript writing, and manuscript preparation. PJ contributed to supervision of study, study design, data analysis, and manuscript revisions. The author(s) read and approved the final manuscript.

\section{Funding}

No funding provided for this study.

\section{Availability of data and materials}

Data is available here: https://wwwn.cdc.gov/nchs/nhanes/ continuousnhanes/overview.aspx?BeginYear=2011.

\section{Declarations}

Ethics approval and consent to participate

This study was exempt from primary author's university Institutional Review Board approval as data was all publicly available and de-identified; hence, data did not meet "human subjects" research definition. Data is available here: https://wwwn.cdc.gov/nchs/nhanes/continuousnhanes/overview. aspx?BeginYear=2011

\section{Consent for publication}

Not applicable.

\section{Competing interests}

The authors declare that they have no competing interests.

\section{Author details}

'Department of Medicine, Rutgers New Jersey Medical School, Newark, NJ, USA. ${ }^{2}$ North American Disease Intervention, Rutgers University, New Brunswick, NJ, USA. ${ }^{3}$ New Jersey Institute of Technology, Newark, NJ, USA. ${ }^{4}$ Division of Cardiology, Department of Medicine, University of Texas Southwestern Medical Center, Dallas, TX, USA.

Received: 5 April 2021 Accepted: 6 December 2021

Published online: 15 January 2022

\section{References}

1. Fryar CD, Ostchega Y, Hales CM, Zhang G, Kruszon-Moran D. Hypertension prevalence and control among adults: United States, 2015-2016. NCHS Data Brief. 2017;289:1-8.

2. Dorans KS, Mills KT, Liu Y, He J. Trends in prevalence and control of hypertension according to the 2017 American College of Cardiology/ American Heart Association (ACC/AHA) guideline. J Am Heart Assoc. 2018;7: e008888.

3. Karppanen H, Mervaala E. Sodium intake and hypertension. Prog Cardiovasc Dis. 2006:49:59-75

4. Sparrenberger F, Cichelero FT, Ascoli AM, Fonseca FP, Weiss G, Berwanger O, et al. Does psychosocial stress cause hypertension? A systematic review of observational studies. J Hum Hypertens. 2009;23:12-9.

5. Rahmouni K, Correia ML, Haynes WG, Mark AL. Obesity-associated hypertension: new insights into mechanisms. Hypertension. 2005:45:9-14.

6. Geleijnse JM, Kok FJ, Grobbee DE. Impact of dietary and lifestyle factors on the prevalence of hypertension in Western populations. Eur J Pub Health. 2004:14:235-9.

7. Franklin SS, Pio JR, Wong ND, Larson MG, Leip EP, Vasan RS, et al. Predictors of new-onset diastolic and systolic hypertension: the Framingham heart study. Circulation. 2005;111:1121-7.

8. Hozawa A, Ohkubo T, Nagai K, Kikuya M, Matsubara M, Tsuji I, et al. Prognosis of isolated systolic and isolated diastolic hypertension as assessed by self-measurement of blood pressure at home: the Ohasama study. Arch Intern Med. 2000;160:3301-6.

9. Visaria A, Lo D. Association between body mass index and hypertension subtypes in Indian and United States adults. Indian Heart J. 2020;72:459-61.

10. Lavie CJ, Milani RV, Ventura HO. Obesity and cardiovascular disease: risk factor, paradox, and impact of weight loss. J Am Coll Cardiol. 2009;53:192532.

11. Kissebah AH, Vydelingum N, Murray R, Evans DJ, Hartz AJ, Kalkhoff RK, et al. Relation of body fat distribution to metabolic complications of obesity. J Clin Endocrinol Metab. 1982;54:254-60.

12. Després JP. Body fat distribution and risk of cardiovascular disease: an update. Circulation. 2012;126:1301-13.

13. Snijder MB, Dekker JM, Visser M, Bouter LM, Stehouwer CD, Yudkin JS, et al. Trunk fat and leg fat have independent and opposite associations with fasting and postload glucose levels: the Hoorn study. Diabetes Care. 2004; 27:372-7

14. Godsland IF, Crook D, Proudler AJ, Stevenson JC. Hemostatic risk factors and insulin sensitivity, regional body fat distribution, and the metabolic syndrome. J Clin Endocrinol Metab. 2005:90:190-7.

15. Wu H, Qi Q, Yu Z, Sun Q, Wang J, Franco OH, et al. Independent and opposite associations of trunk and leg fat depots with adipokines, inflammatory markers, and metabolic syndrome in middle-aged and older Chinese men and women. J Clin Endocrinol Metab. 2010;95:4389-98.

16. Aasen $G$, Fagertun $H$, Halse J. Regional fat mass by DXA: high leg fat mass attenuates the relative risk of insulin resistance and dyslipidaemia in obese but not in overweight postmenopausal women. Scand J Clin Lab Invest. 2008;68:204-11.

17. Zhu $Y$, Lin $Q$, Zhang $Y$, Deng $H, H u X$, Yang $X$, et al. Mid-upper arm circumference as a simple tool for identifying central obesity and insulin resistance in type 2 diabetes. PLoS One. 2020:15:e0231308.

18. Sakai $Y$, Ito H, Egami Y, Ohoto N, Hijii C, Yanagawa M, et al. Favourable association of leg fat with cardiovascular risk factors. J Intern Med. 2005;257: 194-200.

19. Zhang X, Hu EA, Wu H, Malik V, Sun Q. Associations of leg fat accumulation with adiposity-related biological factors and risk of metabolic syndrome. Obesity (Silver Spring). 2013:21:824-30.

20. Hu G, Bouchard C, Bray GA, Greenway FL, Johnson WD, Newton RL Jr, et al. Trunk versus extremity adiposity and cardiometabolic risk factors in white and African American adults. Diabetes Care. 2011;34:1415-8.

21. Williams MJ, Hunter GR, Kekes-Szabo T, Snyder S, Treuth MS. Regional fat distribution in women and risk of cardiovascular disease. Am J Clin Nutr. 1997;65:855-60

22. Tankó LB, Bagger YZ, Alexandersen P, Larsen PJ, Christiansen C. Peripheral adiposity exhibits an independent dominant antiatherogenic effect in elderly women. Circulation. 2003;107:1626-31.

23. Heitmann BL, Frederiksen P. Thigh circumference and risk of heart disease and premature death: prospective cohort study. BMJ. 2009:339:b3292.

24. Lee M, Choh AC, Demerath EW, Towne B, Siervogel RM, Czerwinski SA. Associations between trunk, leg and total body adiposity with arterial stiffness. Am J Hypertens. 2012;25:1131-7.

25. Han E, Lee YH, Lee BW, Kang ES, Lee IK, Cha BS. Anatomic fat depots and cardiovascular risk: a focus on the leg fat using nationwide surveys (KNHA NES 2008-2011). Cardiovasc Diabetol. 2017:16:54

26. Zong G, Zhang Z, Yang Q, Wu H, Hu FB, Sun Q. Total and regional adiposity measured by dual-energy $X$-ray absorptiometry and mortality in NHANES 1999-2006. Obesity (Silver Spring). 2016;24:2414-21.

27. Koh H, Hayashi T, Sato KK, Harita N, Maeda I, Nishizawa Y, et al. Visceral adiposity, not abdominal subcutaneous fat area, is associated with high blood pressure in Japanese men: the Ohtori study. Hypertens Res. 2011;34: 565-72.

28. Kotchen TA. Obesity-related hypertension: epidemiology, pathophysiology, and clinical management. Am J Hypertens. 2010;23:1170-8.

29. Zhang Y, Somers KR, Becari C, Polonis K, Pfeifer MA, Allen AM, et al. Comparative expression of renin-angiotensin pathway proteins in visceral versus subcutaneous fat. Front Physiol. 2018;9:1370.

30. Hillebrand S, de Mutsert R, Christen T, Maan AC, Jukema JW, Lamb HJ, et al. Body fat, especially visceral fat, is associated with electrocardiographic measures of sympathetic activation. Obesity (Silver Spring). 2014;22:1553-9.

31. Yan S, Zhao X, Shen $X$, Yang L, Yuan $X$, Huang $L$, et al. Abnormal regional body fat distribution also exists in non-obese subjects with high blood pressure. Clin Exp Hypertens. 2013;35:528-33. 
32. Kouda K, Nakamura H, Fujita Y, Ohara K, Iki M. Increased ratio of trunk to appendicular fat and increased blood pressure: study of a general population of Hamamatsu children. Circ J. 2012;76:2848-54.

33. Centers for Disease Control and Prevention. National Health and Nutrition Examination Survey: NHANES survey methods and analytic guidelines. 2018. https://wwwn.cdc.gov/nchs/nhanes/AnalyticGuidelines.aspx. Accessed 1 Apr 2021.

34. Centers for Disease Control and Prevention. National Health and Nutrition Examination Survey (NHANES): Body composition procedures manual. 2013. https://www.cdc.gov/nchs/data/nhanes/nhanes_13_14/2013_Body_ Composition_DXA.pdf. Accessed 4 Nov 2020.

35. Whelton PK, Carey RM, Aronow WS, Casey DE Jr, Collins KJ, Dennison Himmelfarb C, et al. 2017 ACC/AHA/AAPA/ABC/ACPM/AGS/APhA/ASH/ ASPC/NMA/PCNA guideline for the prevention, detection, evaluation, and management of high blood pressure in adults: a report of the American College of Cardiology/American Heart Association Task Force on Clinical Practice Guidelines. J Am Coll Cardiol. 2018;71:e127-248.

36. Centers for Disease Control and Prevention. National Health and Nutrition Examination Survey (NHANES): MEC laboratory procedures manual. Hyattsville: National Center for Health Statistics; 2013.

37. Kienitz T, Quinkler M. Testosterone and blood pressure regulation. Kidney Blood Press Res. 2008:31:71-9.

38. Park SY, Kwon KY, Kim JH, Choi HH, Han KH, Han JH. Association between appendicular fat mass and metabolic risk factors. Korean J Fam Med. 2014; 35:182-9.

\section{Publisher's Note}

Springer Nature remains neutral with regard to jurisdictional claims in published maps and institutional affiliations.

Ready to submit your research? Choose BMC and benefit from:

- fast, convenient online submission

- thorough peer review by experienced researchers in your field

- rapid publication on acceptance

- support for research data, including large and complex data types

- gold Open Access which fosters wider collaboration and increased citations

- maximum visibility for your research: over $100 \mathrm{M}$ website views per year

At $\mathrm{BMC}$, research is always in progress.

Learn more biomedcentral.com/submissions 